\title{
Inhibition and Stimulation of Different Dechlorinating Bacterial Cultures Using Iron Particles
}

\author{
Dorothea Summer, Philipp Schöftner, Bernhard Wimmer, Thomas G. Reichenauer \\ Austrian Institute of Technology GmbH, Energy Department, Environmental Resources \& Technologies \\ Konrad-Lorenz-Strasse 24, 3430 Tulln, Austria \\ dorothea.summer@ait.ac.at; philipp.s@posteo.de
}

\section{Extended Abstract}

At about one third of severely contaminated sites in Europe chlorinated ethenes like perchloroethene (PCE) can be found. Due to its high persistency, it leads to long lasting pollutions and forms long groundwater plumes. PCE reaches the environment through anthropogenic sources, such as dry-cleanings and metal-working industries. Therefore PCE contaminations can mainly be found in urban regions and consequently in many cases only in-situ ground water remediation methods can be used for clean-up.

This study aims to combine chemical and biological in-situ remediation methods to increase their efficiency by eliminating their shortcomings. In the chemical degradation process zero-valent iron (ZVI) is used to reductively dechlorinate PCE-molecules. But ZVI is also consumed by anaerobic corrosion producing hydrogen [1]-[3]. Thus, an excess of ZVI particles has to be used to accomplish a successful clean-up, which increases the costs of the remediation drastically. To achieve biological degradation strictly anaerobic strains of the bacteria Dehalococcoides are used, which are able to completely dechlorinate PCE to ethene by utilizing hydrogen as an electron donor [4]-[6]. These bacteria can occur naturally at contaminated sites or can also be injected into the groundwater. The dehalogenating bacteria compete for the existing hydrogen with other anaerobic bacteria, which are often more successful in receiving electrons from the donor [7], [8]. A depletion in hydrogen often leads to an incomplete microbial degradation and an accumulation of even more hazardous metabolites, such as vinyl chloride. By combining the two processes the hydrogen, produced during the anaerobic corrosion of ZVI, could be used by the bacteria for further PCE degradation. This way the remediation time, the amount of ZVI and consequently also the remediation costs could be reduced.

In a first step PCE degradation experiments in microcosms were conducted. The degradation with different ZVI particles (nano- and micro-scaled), dechlorinating cultures and their combinations were compared. The microcosms were prepared in an anaerobic chamber to ensure optimal conditions for the strictly anaerobic bacteria. HEPES and $\mathrm{Na}_{2} \mathrm{~S}$ were used to ensure stable $\mathrm{pH}$ and redox conditions. Molasses was added as an economic carbon source. PCE, its metabolites and end products were measured with GC-FID and hydrogen with GC-TCD. $\mathrm{pH}$ and redox conditions were checked over the course of the experiments.

The two cultures investigated showed different behaviours: one could completely degrade PCE to ethene (culture A), while the other led to an intermediate accumulation of cis-DCE and a slower development of ethene (culture B). Correspondingly also the combination with the different iron particles showed different results. We could achieve a stimulation of the complete microbial dehalogenation with culture B with the used iron particles. In both cases the nanoscaled ZVI even slowed down the conversion of PCE to TCE. The addition of ZVI could reduce the accumulation of unwanted metabolites in the microcosms with both cultures. The hydrogen produced by the iron particles was used by both cultures, but not only for dechlorination.

\section{References}

[1] J. Filip et al., "Anaerobic reaction of nanoscale zerovalent iron with water: Mechanism and kinetics," J. Phys. Chem. $C$, vol. 118 , no. 25 , pp. $13817-13825,2014$.

[2] A. B. Cundy, L. Hopkinson, and R. L. D. Whitby, "Use of iron-based technologies in contaminated land and groundwater remediation: A review," Sci. Total Environ., vol. 400, no. 1-3, pp. 42-51, 2008.

[3] N. C. Mueller et al., "Application of nanoscale zero valent iron (NZVI) for groundwater remediation in Europe," 
Environ. Sci. Pollut. Res., vol. 19, no. 2, pp. 550-558, 2012.

[4] J. A. Field and R. Sierra-Alvarez, "Biodegradability of chlorinated solvents and related chlorinated aliphatic compounds," Rev. Environ. Sci. Biotechnol., vol. 3, no. 3, pp. 185-254, 2004.

[5] I. Kranzioch et al., "Dechlorination and organohalide-respiring bacteria dynamics in sediment samples of the Yangtze Three Gorges Reservoir," Environ. Sci. Pollut. Res., vol. 20, no. 10, pp. 7046-7056, 2013.

[6] X. Maymó-Gatell, Y. T. Chien, J. M. Gossett, and S. H. Zinder, "Isolation of a bacterium that reductively dechlorinates tetrachloroethene to ethene," Science (80-. ), vol. 276, no. 5318, pp. 1568-1571, 1997.

[7] C. R. Smatlak, J. M. Gossett, and S. H. Zinder, "Comparative kinetics of hydrogen utilization for reductive dechlorination of tetrachloroethene and methanogenesis in an anaerobic enrichment culture," Environ. Sci. Technol., vol. 30, no. 9, pp. 2850-2858, 1996.

[8] Y. Yang and P. L. McCarty, "Competition for hydrogen within a chlorinated solvent dehalogenating anaerobic mixed culture,” Environ. Sci. Technol., vol. 32, no. 22, pp. 3591-3597, 1998. 